\title{
QUADRATIC LIE CONFORMAL SUPERALGEBRAS RELATED TO NOVIKOV SUPERALGEBRAS
}

\author{
P.S. KOLESNIKOV ${ }^{1)}$, R.A. KOZLOV ${ }^{1) 2}$, A.S. PANASENKO ${ }^{12)}$
}

\begin{abstract}
We study quadratic Lie conformal superalgebras associated with Novikov superalgebras. For every Novikov superalgebra $(V, \circ)$, we construct an enveloping differential Poisson superalgebra $U(V)$ with a derivation $d$ such that $u \circ v=u d(v)$ and $\{u, v\}=u \circ v-(-1)^{|u||v|} v \circ u$ for $u, v \in V$. The latter means that the commutator Gelfand-Dorfman superalgebra of $V$ is special. Next, we prove that every quadratic Lie conformal superalgebra constructed on a finitedimensional special Gelfand-Dorfman superalgebra has a finite faithful conformal representation. This statement is a step toward a solution of the following open problem: whether a finite Lie conformal (super)algebra has a finite faithful conformal representation.
\end{abstract}

\section{INTRODUCTION}

Novikov algebras appeared in [11] as a class of algebras giving rise to Hamiltonian operators in the formal calculus of variations. Independently, these algebras were introduced in [4] as a tool for studying linear Poisson brackets of hydrodynamic type. The study of structure theory of Novikov algebras was initiated in [30], significant progress in this direction was obtained in [1, 17, 26, 28].

A class of more complicated structures called Gelfand-Dorfman bialgebras [27] was also introduced in [11] as a source of Hamiltonian operators. A GelfandDorfman bialgebra is a linear space with two bilinear operations $(\cdot \circ \cdot)$ and $[\cdot, \cdot]$, where $(\cdot \circ \cdot)$ is a Novikov product (left symmetric, right commutative), i.e.,

$$
\begin{aligned}
\left(x_{1} \circ x_{2}\right) \circ x_{3}-x_{1} \circ\left(x_{2} \circ x_{3}\right) & =\left(x_{2} \circ x_{1}\right) \circ x_{3}-x_{2} \circ\left(x_{1} \circ x_{3}\right), \\
\left(x_{1} \circ x_{2}\right) \circ x_{3} & =\left(x_{1} \circ x_{3}\right) \circ x_{2},
\end{aligned}
$$

$[\cdot, \cdot]$ is a Lie product, and the following compatibility relation holds:

$$
\left[x_{1}, x_{2} \circ x_{3}\right]-\left[x_{3}, x_{2} \circ x_{1}\right]+\left[x_{2}, x_{1}\right] \circ x_{3}-\left[x_{2}, x_{3}\right] \circ x_{1}-x_{2} \circ\left[x_{1}, x_{3}\right]=0 .
$$

In order to avoid a confusion with the well-known notion of a bialgebra as an algebra equipped with a coproduct, we will use the term GD-algebra ([17]) for a GelfandDorfman bialgebra.

2010 Mathematics Subject Classification. 17B69 17B63, 37K30,

Key words and phrases. Poisson superalgebra, Novikov superalgebra, Gelfand-Dorfman superalgebra, conformal superalgebra.

The work is supported by Mathematical Center in Akademgorodok under agreement No. 07515-2019-1613 with the Ministry of Science and Higher Education of the Russian Federation. 
Novikov algebras and GD-algebras play an important role in the combinatorics of differential algebras (see [17, Theorem 7]). Namely, the identities that hold for the operations

$$
a \succ b=d(a) \cdot b, \quad a \prec b=a \cdot d(b),
$$

$a, b \in A$, with $A$ being an algebra in a multilinear variety Var, may be calculated by means of the Manin white product [12] of the operad Var and the operads Nov and GD!, the Koszul dual of GD.

Every differential Poisson algebra gives rise to a GD-algebra [29]: if $P$ is a commutative differential algebra with a derivation $d$ equipped with a Poisson bracket $\{\cdot, \cdot\}$ such that $d$ acts as a derivation relative to $\{\cdot, \cdot\}$ then $P$ is a GD-algebra with operations $x \circ y=z d(y),[x, y]=\{x, y\}, x, y \in P$. GD-algebras that embed into Poisson differential algebras in this way are called special [17]. Not all GD-algebras are special, a series of necessary conditions for a GD-algebra to be special was found in the last paper. Below, we will state some precise examples of non-special GD-algebras.

It was noted in [11, Remark 6.3] that if we enrich a Novikov algebra $(V, \circ)$ with the operation $[x, y]=x \circ y-y \circ x, x, y \in V$, then we obtain a GD-algebra. It is not hard to check that such a system obtained from a Novikov algebra meets necessary conditions of speciality found in [17]. In Section 2, we prove that all GDsuperalgebras arising from Novikov superalgebras relative to (super-)commutator are indeed special, and we construct an enveloping differential Poisson superalgebra for every Novikov algebra.

It turned out (see [13, 27]) that GD-algebras are in one-to-one correspondence with quadratic Lie conformal algebras. The latter structures appeared in [14] as a tool in the study of vertex operator algebras. Conformal algebras and their generalizations (pseudo-algebras) also turn to be useful for the classification of Poisson brackets of hydrodynamic type [2]. A conformal algebra is a module $C$ over the polynomial algebra $H=\mathbb{k}[\partial]$ equipped with a "multi-valued" operation $C \otimes C \rightarrow C[\lambda]$, i.e., a product of two elements from $C$ is a polynomial in a formal variable $\lambda$ with coefficients in $C$. The axioms defining a conformal algebra are stated in Section 3 .

One of the most intriguing questions in the theory of conformal algebras is motivated by the Ado theorem: Does a Lie conformal algebra which is a finitely generated free module over $H$ have a faithful representation on a finitely generated free $H$ module? (The condition of freeness is necessary: in conformal algebras and their modules, every torsion element belongs to the corresponding annihilator.) In the case of positive answer, we may faithfully represent every Lie conformal algebra with polynomial matrices in $\operatorname{Cend}_{n}$, see [6]. By now, the most general result in this direction says that if a Lie conformal algebra as above has a Levi decomposition (i.e., its solvable radical splits) then it has a finite faithful representation (FFR).

Therefore, one may observe close relations between Novikov algebras, Poisson algebras, and conformal algebras. In Section 4, we prove that every quadratic Lie 
conformal superalgebra obtained from a special GD-algebra has a FFR. As an application, every quadratic Lie conformal superalgebra obtained from a Novikov superalgebra has a FFR.

\section{Gelfand-Dorfman SUPERALGEBRAS}

A $\mathbb{Z}_{2}$-graded space $V=V_{0} \oplus V_{1}$ with two bilinear operations $(\cdot \circ \cdot),[\cdot, \cdot]: V \otimes V \rightarrow V$ which respect the grading is said to be a GD-superalgebra if

- $V$ is a Novikov superalgebra relative to $(\cdot \circ \cdot)$;

- $V$ is a Lie superalgebra relative to $[\cdot, \cdot]$;

- for every homogeneous $a, b, c \in V$

$$
[a \circ b, c]-a \circ[b, c]+[a, b] \circ c+(-1)^{|a||b|}[b, a \circ c]-(-1)^{|b||c|}[a, c] \circ b=0 .
$$

A series of examples of GD-superalgebras may be obtained from differential Poisson superalgebras. Let $P=P_{0} \oplus P_{1}$ be an associative and commutative superalgebra equipped with an operation $\{\cdot, \cdot\}$ such that

$$
\begin{gathered}
\left\{P_{i}, P_{j}\right\} \subseteq P_{(i+j)} \quad(\bmod 2), \quad\{a, b\}=-(-1)^{|a||b|}\{b, a\} \\
\{a,\{b, c\}\}-(-1)^{|a||b|}\{b,\{a, c\}\}=\{\{a, b\}, c\}, \\
\{a, b c\}=\{a, b\} c+(-1)^{|a||b|} b\{a, c\},
\end{gathered}
$$

for all homogeneous $a, b, c \in P$. Then $P$ is called a Poisson superalgebra.

Suppose a Poisson superalgebra $P$ has an (even) derivation $d: P \rightarrow P$, i.e.,

$$
d\left(P_{i}\right) \subseteq P_{i}, \quad d(a b)=d(a) b+a d(b), \quad d\{a, b\}=\{d(a), b\}+\{a, d(b)\},
$$

for all $a, b \in P$. Then the same space $P$ equipped with

$$
a \circ b=a d(b), \quad[a, b]=\{a, b\}
$$

is a GD-superalgebra [27, Theorem 3.2] denoted $P^{(d)}$.

For a GD-superalgebra $V$, we say $V$ is special if there exists a Poisson superalgebra $P$ with a derivation $d$ such that $V \subseteq P^{(d)}$. Non-special GD-superalgebras are said to be exceptional.

Exceptional GD-superalgebras exist: it was shown in [17] by means of implicit computational arguments. Let us state below an explicit example of a 3-dimensional exceptional GD-algebra.

Example 1. Let $V$ be a 3-dimensional space with a basis $\{x, y, z\}$ equipped with a Lie algebra structure

$$
[x, y]=z, \quad[x, z]=[y, z]=0
$$

(Heisenberg Lie algebra). It is straightforward to check that the operation $(\cdot \circ \cdot)$ on $V$ given by

$$
\begin{gathered}
x \circ x=x-y, \quad y \circ x=-x \circ y=y, \\
x \circ z=z \circ x=y \circ z=z \circ y=y \circ y=z \circ z=0
\end{gathered}
$$


turns $V$ into a GD-algebra. If we assume $V$ to be special, then there exists a Poisson differential algebra $P$ such that $V \subset P^{(d)}$. Consider the expression $\left\{x, x^{\prime}\right\} x x^{\prime} \in P$ (here $x^{\prime}$ stands for $d(x)$ ). On the one hand,

$$
\begin{aligned}
\left\{x, x^{\prime}\right\}\left(x x^{\prime}\right)=\left\{x, x^{\prime}\right\}(x \circ x)=\left\{x,(x \circ x) x^{\prime}\right\}-\{x, x \circ x\} x^{\prime} & \\
& =[x,(x \circ x) \circ x]-[x, x \circ x] \circ x=-2 z ;
\end{aligned}
$$

on the other hand,

$$
\left\{x, x^{\prime}\right\}\left(x x^{\prime}\right)=\left(\left\{x, x^{\prime}\right\} x\right) x^{\prime}=\left\{x, x x^{\prime}\right\} x^{\prime}=[x, x \circ x] \circ x=0 .
$$

Hence, $V$ cannot be embedded into a differential Poisson algebra.

Another series of examples was proposed in [11] for the non-graded case. In general, if $V$ is a Novikov superalgebra then the operation

$$
[a, b]=(a \circ b)-(-1)^{|a||b|}(b \circ a), \quad a, b \in V_{0} \cup V_{1},
$$

turns $V$ into a GD-superalgebra denoted $V^{(-)}$. It was conjectured in [17] that for every Novikov algebra the commutator GD-algebra $V^{(-)}$is special. In this section, we prove the conjecture in the $\mathbb{Z}_{2}$-graded setting.

In the following, we will need the embedding theorem proved in [5] for Novikov algebras and then in [31] for Novikov superalgebras (in these two papers, the term "Gelfand-Dorfman-Novikov algebra" is used for what we call "Novikov algebra" following the common terminology proposed in [22]).

Theorem 1 ([5, Theorem 3], [31, Theorem 3.8]). For every Novikov (super)algebra $(V, \circ)$ there exists an associative and (super)commutative algebra $A$ with an even derivation d such that $V \subset A$ and $u \circ v=u d(v)$ for $u, v \in V$.

In particular, the universal enveloping differential algebra of a given Novikov superalgebra $V=V_{0} \oplus V_{1}$ may be constructed as follows. Let $X=X_{0} \cup X_{1}$ be a linear basis of $V$, where $X_{i}$ is a basis of $V_{i}, i=0,1$. Denote by $s \operatorname{ComDer}\left\langle X_{0} \cup X_{1}, d\right\rangle$ the free associative supercommutative differential algebra generated by even variables $X_{0}$ and odd variables $X_{1}$ with an even derivation $d$. Apparently, $s \operatorname{ComDer}\left\langle X_{0} \cup X_{1}, d\right\rangle \simeq$ $\mathbb{k}\left[d^{\omega} X_{0}\right] \otimes \bigwedge\left(\mathbb{k} d^{\omega} X_{1}\right)$, where

$$
d^{\omega} X_{i}=\left\{d^{n}(x) \mid n \geq 0, x \in X_{i}\right\} .
$$

Consider the (differential) ideal $I_{V}$ of $s \operatorname{ComDer}\left\langle X_{0} \cup X_{1}, d\right\rangle$ generated by $u(x, y)=$ $x d(y)-x \circ y, x, y \in X$ (here $x \circ y$ stands for the linear form in $\mathbb{k} X$ representing the Novikov product in $V)$. As a non-differential ideal of $\mathbb{k}\left[d^{\omega} X_{0}\right] \otimes \wedge\left(\mathbb{k} d^{\omega} X_{1}\right), I_{V}$ is generated by all derivatives of $u(x, y)$. Theorem 1 implies $I_{V} \cap \mathbb{k} X=0$.

In order to define a (super) Poisson bracket $\{\cdot, \cdot\}$ on $s \operatorname{ComDer}\left\langle X_{0} \cup X_{1}, d\right\rangle$ it is enough to determine polynomials $\left\{d^{n}(x), d^{m}(y)\right\}$ for $x, y \in X, n, m \geq 0$, and then extend the bracket in a unique way to the entire $s \operatorname{ComDer}\left\langle X_{0} \cup X_{1}, d\right\rangle$ by the Leibniz rule (6). If the bracket respects $\mathbb{Z}_{2}$-grading and is (super) anti-commutative on the generators from $d^{\omega} X$ then so is its extension. In order to simplify notations, let us denote $d^{n}(x)$ by $x^{(n)}$ for $x \in X, n \geq 0$. 
Lemma 1. Let $\{\cdot, \cdot\}$ be a bracket on $s \operatorname{ComDer}\left\langle X_{0} \cup X_{1}, d\right\rangle$ obtained by expanding

$$
\left\{x^{(m)}, y^{(n)}\right\}=(n-1) x^{(m+1)} y^{(n)}-(m-1) x^{(m)} y^{(n+1)}, \quad x, y \in X, n, m \geq 0 .
$$

Then $\{\cdot, \cdot\}$ satisfies the Jacobi identity (5)).

Proof. For $x, y, z \in X, n, m, k \geq 0$, evaluate

$$
\begin{aligned}
&\left\{x^{(m)}\left\{y^{(n)}, z^{(k)}\right\}\right.=\left\{x^{(m)},(k-1) y^{(n+1)} z^{(k)}-(n-1) y^{(n)} z^{(k+1)}\right\} \\
&=(k-1)\left(\left\{x^{(m)}, y^{(n+1)} z^{(k)}+(-1)^{|x||y|} y^{(n+1)}\left\{x^{(m)}, z^{(k)}\right\}\right\}\right) \\
&-(n-1)\left(\left\{x^{(m)}, y^{(n)}\right\} z^{(k+1)}+(-1)^{|x||y|} y^{(n)}\left\{x^{(m)}, z^{(k+1)}\right\}\right) \\
&=(k-1)\left(\left(n x^{(m+1)} y^{(n+1)}-(m-1) x^{(m)} y^{(n+2)}\right) z^{(k)}\right. \\
&+\left.y^{(n+1)}(-1)^{|x||y|}\left((k-1) x^{(m+1)} z^{(k)}-(m-1) x^{(m)} z^{(k+1)}\right)\right) \\
&-(n-1)\left(\left((n-1) x^{(m+1)} y^{(n)}-(m-1) x^{(m)} y^{(n+1)}\right) z^{(k)}\right. \\
&\left.\quad-(-1)^{|x||y|} y^{(n)}\left(k x^{(m)} z^{(k+1)}-(m-1) x^{(m)} z^{(k+2)}\right)\right) \\
&=(k-1)(n+k-1) x^{(m+1)} y^{(n+1)} z^{(k)}-(k-1)(m-1) x^{(m)} y^{(n+2)} z^{(k)} \\
&+(m-1)(n-k) x^{(m)} y^{(n+1)} z^{(k)}-(n-1)(k+n-1) x^{(m+1)} y^{(n)} z^{(k+1)} \\
& \quad+(n-1)(m-1) x^{(m)} y^{(n)} z^{(k+2)} .
\end{aligned}
$$

The Jacobi identity (5) is equivalent to

$$
\begin{aligned}
(-1)^{|x||z|}\left\{x^{(m)},\left\{y^{(n)}, z^{(k)}\right\}\right\}+(-1)^{|y||x|}\left\{y^{(n)},\left\{z^{(k)}, x^{(m)}\right\}\right\} & \\
& +(-1)^{|y||z|}\left\{z^{(k)},\left\{x^{(m)}, y^{(n)}\right\}\right\}=0
\end{aligned}
$$

which is easy to check by the cyclic permutation of variables in (9).

Lemma 2. The operation $d$ on $s \operatorname{ComDer}\left\langle X_{0} \cup X_{1}, d\right\rangle$ is a derivation relative to the bracket from Lemma 1 .

Proof. It is enough to check that

$$
\begin{gathered}
d\left\{x^{(m)}, y^{(n)}\right\}=(n-1)\left(x^{(m+2)} y^{(n)}+x^{(m+1)} y^{(n+1)}\right)-(m-1)\left(x^{(m)} x^{(n+2)}+x^{(m+1)} y^{(n+1)}\right) \\
=(n-1) x^{(m+2)} y^{(n)}-m x^{(m+1)} y^{(n+1)}+x^{(m+1)} y^{(n+1)}+n x^{(m+1)} y^{(n+1)} \\
-(m-1) x^{(m+1)} y^{(n+1)}-x^{(m+1)} y^{(n+1)}=\left\{x^{(m+1)}, y^{(n)}\right\}+\left\{x^{(m)}, y^{(n+1)}\right\} \\
=\left\{d x^{(m)}, y^{(n)}\right\}+\left\{x^{(m)}, d y^{(n)}\right\}
\end{gathered}
$$

for $x, y \in X, n, m \geq 0$. Since the bracket on the entire $s \operatorname{ComDer}\left\langle X_{0} \cup X_{1}, d\right\rangle$ is calculated via the Leibniz rule, we have

$$
d\{f, g\}=\{d(f), g\}+\{f, d(g)\}
$$

for all $f, g \in s \operatorname{ComDer}\left\langle X_{0} \cup X_{1}, d\right\rangle$.

Lemma 3. The ideal $I_{V}$ is invariant under all operations $\{f, \cdot\}, f \in s \operatorname{ComDer}\left\langle X_{0} \cup\right.$ $\left.X_{1}, d\right\rangle$, where $\{\cdot, \cdot\}$ is the bracket from Lemma 1 . 
Proof. Again, since $\{f, \cdot\}$ is defined via the Leibniz rule, it is enough to consider $f=z^{(n)}, z \in X, n \geq 0$. Moreover, Lemma 2 implies

$$
\left\{z^{(n)}, \cdot\right\}=d\left\{z^{(n-1)}, \cdot\right\}-\left\{z^{(n-1)}, d(\cdot)\right\},
$$

so it is enough to check the invariance of $I_{V}$ under $\{z, \cdot\}$, where $z=z^{(0)}$. The Leibniz rule (6) and Lemma 2 show that it is enough to verify $\{z, u(x, y)\} \in I_{V}$ for all $x, y, z \in X$. Indeed,

$$
\begin{gathered}
\{z, u(x, y)\}=\left\{z, x y^{\prime}\right\}-\{z, x \circ y\}=\{z, x\} y^{\prime}+(-1)^{|x||z|} x\left\{z, y^{\prime}\right\}+z^{\prime}(x \circ y)-z d(x \circ y) \\
=\left(z x^{\prime}-z^{\prime} x\right) y^{\prime}+(-1)^{|x||z|} x z y^{\prime \prime}-z\left(x y^{\prime}\right)^{\prime}+z^{\prime} x y^{\prime} \\
=z x^{\prime} y^{\prime}-z^{\prime} x y^{\prime}+z x y^{\prime \prime}-z x y^{\prime \prime}-z x^{\prime} y^{\prime}+z^{\prime} x y^{\prime}=0 .
\end{gathered}
$$

Hence, the entire differential ideal $I_{V}$ generated by $u(x, y)$ is invariant under the bracket defined by (8).

Theorem 2. Let $V$ be a Novikov superalgebra. Then the GD-algebra $V^{(-)}$is special.

Proof. Lemmas 1, 2, 3 show the quotient $U(V)=s \operatorname{ComDer}\left\langle X_{0} \cup X_{1}, d\right\rangle / I_{V}$ is a differential Poisson algebra. Theorem 1 guaranties that $V$ embeds into $U(V)$ with $u \circ v=u d(v), u, v \in V$. Moreover, (8) implies $\{x, y\}=-x^{\prime} y+x y^{\prime}=x \circ y-$ $(-1)^{|x||y|} y \circ x$ for $x, y \in X$. Therefore, $U(V)$ is a differential Poisson enveloping superalgebra of $V^{(-)}$.

\section{Conformal superalgebras}

In this section we recall the main definitions concerning Lie conformal (super)algebras and their relations to GD-(super)algebras.

Let $L$ be a Lie superalgebra over a field $\mathbb{k}$ of characteristic zero and let $H=\mathbb{k}[\partial]$ be the algebra of polynomials in one variable.

Two formal distributions $a(z), b(z) \in L\left[\left[z, z^{-1}\right]\right]$ are said to be mutually local if there exists $N \geq 0$ such that

$$
[a(w), b(z)](w-z)^{N}=0 \in L\left[\left[z, z^{-1}, w, w^{-1}\right]\right] .
$$

For a pair of mutually local formal distributions $a(z), b(z)$ their product $[a(w), b(z)]$ may be uniquely presented as a finite distribution

$$
[a(w), b(z)]=\sum_{n=0}^{N-1} c_{n}(z) \frac{1}{n !} \frac{\partial^{n} \delta(w-z)}{\partial z^{n}},
$$

where $c_{n}(z) \in L\left[\left[z, z^{-1}\right]\right], \delta(w-z)=\sum_{s \in \mathbb{Z}} w^{s} z^{-s-1}$ is the formal delta-function.

The collection of formal distributions $c_{n}(z), n=0,1, \ldots, N-1$, associated to a given pair $a(z), b(z)$ may be written as a polynomial in a new formal variable $\lambda$ :

$$
\left[a(z)_{(\lambda)} b(z)\right]=\sum_{n=0}^{N-1} \frac{\lambda^{n}}{n !} c_{n}(z) \in\left(L\left[\left[z, z^{-1}\right]\right]\right)[\lambda] .
$$


The latter space may be identified with $\mathbb{k}[\partial, \lambda] \otimes_{H} L\left[\left[z, z^{-1}\right]\right]$, where $\partial$ acts on $L\left[\left[z, z^{-1}\right]\right]$ as the ordinary derivation with respect to $z$.

An $H$-invariant subspace $C$ of $L\left[\left[z, z^{-1}\right]\right]$ which consists of pairwize mutually local distributions and is closed with respect to the operation $\left[\cdot{ }_{(\lambda)} \cdot \cdot\right]$ (i.e., $a, b \in C$ implies $\left.\left[a_{(\lambda)} b\right] \in C[\lambda]\right)$ provides an example of the following class of algebraic structures.

Definition 1 ([14, Chapter 2]). A $\mathbb{Z}_{2}$-graded $H$-module $C=C_{0} \oplus C_{1}$ equipped with a linear map $(\lambda$-bracket)

$$
\left[\cdot{ }_{(\lambda)} \cdot\right]: C \otimes C \rightarrow \mathbb{k}[\partial, \lambda] \otimes_{H} C \simeq C[\lambda]
$$

is called a Lie conformal superalgebra if $\left[C_{i(\lambda)} C_{j}\right] \subseteq C_{(i+j)(\bmod 2)}[\lambda]$ and

$$
\begin{gathered}
{\left[\partial x_{(\lambda)} y\right]=-\lambda\left[x_{(\lambda)} y\right]} \\
{\left[x_{(\lambda)} \partial y\right]=(\partial+\lambda)\left[x_{(\lambda)} y\right]} \\
{\left[x_{(\lambda)} y\right]=(-1)^{|x||y|}\left[y_{(-\partial-\lambda)} x\right]} \\
\left.\left[x_{(\lambda)}\left[y_{(\mu)} z\right]\right]-(-1)^{|x||y|}\left[y_{(\mu)}\left[x_{(\lambda)} z\right]\right]=\left[x_{(\lambda)} y\right]_{(\lambda+\mu)} z\right]
\end{gathered}
$$

for all $x, y \in C$. Here $|x|$ stands for the parity of a homogeneous element $x \in C_{0} \cup C_{1}$.

An operation $[\cdot(\lambda) \cdot]$ satisfying (10) and (11) is said to be sesqui-linear.

In particular, if $C$ is a free $H$-module with a basis $B$ then the $\lambda$-bracket on $C$ is uniquely determined by polynomials $p_{x, y}^{z} \in \mathbb{k}[\partial, \lambda], x, y, z \in B$, so that

$$
\left[x_{(\lambda)} y\right]=\sum_{z \in B} p_{x, y}^{z}(\partial, \lambda) z .
$$

Example 2 ([27]). Let $V$ be a GD-superalgebra. Then the space of Laurent polynomials $V\left[t, t^{-1}\right]$ is a Lie superalgebra relative to the bracket

$$
\left[a t^{n}, b t^{m}\right]=[a, b] t^{n+m}+n(a \circ b) t^{n+m-1}-(-1)^{|a||b|}(b \circ a) t^{n+m-1}, \quad a, b \in V .
$$

For $a \in V$, let $a(z)$ stand for the formal distribution

$$
a(z)=\sum_{n \in \mathbb{Z}} a t^{n} z^{-n-1} \in\left(V\left[t, t^{-1}\right]\right)\left[\left[z, z^{-1}\right]\right] .
$$

The linear span of all such formal distributions and all their derivatives (with respect to $z$ ) is a Lie conformal superalgebra $L(V)$ isomorphic to $H \otimes V$ relative to the $\lambda$ bracket

$$
\left[a_{(\lambda)} b\right]=[a, b]+(-1)^{|a||b|}(\partial+\lambda)(b \circ a)+\lambda(a \circ b), \quad a, b \in V .
$$

Conformal superalgebras obtained from GD-superalgebras as in Example 2 are called quadratic Lie conformal superalgebras. As shown in [27, Theorem 2.2], every Lie conformal superalgebra structure on a free $H$-module $L=H \otimes V$ given by linear polynomials $p_{x, y}^{z}(\partial, \lambda), x, y, z \in V$, is quadratic.

An important particular example of a quadratic Lie conformal algebra comes from the 1-dimensional GD-algebra $V=\mathbb{k} v$ with $v \circ v=v$ :

$$
[v(\lambda) v]=\partial v+2 \lambda v
$$


In this case, $L(V)$ is the Virasoro conformal algebra.

Suppose $V$ and $W$ are two $H$-modules. The space of all linear maps

$$
\alpha: V \rightarrow W[\lambda], \quad v \mapsto \alpha_{\lambda}(v),
$$

such that $\alpha_{\lambda}(\partial v)=(\partial+\lambda) \alpha_{\lambda}(v)$ for all $v \in V$ is denoted by $\operatorname{Chom}(V, W)$ (the space of conformal homomorphisms from $V$ to $W$ ) [14]. If $V=W$ then $\operatorname{Chom}(V, W)$ is denoted by Cend $V$.

If $V$ is a finitely generated $H$-module then the operation

$$
(\cdot \lambda \cdot): \text { Cend } V \otimes \text { Cend } V \rightarrow \text { Cend } V[\lambda]
$$

given by

$$
\left(\alpha_{(\lambda)} \beta\right)_{\mu}(v)=\alpha_{\lambda}\left(\beta_{\mu-\lambda}(v)\right), \quad v \in V
$$

satisfies (10) and (11). Note that if $V$ is not a finitely generated $H$-module then we cannot say in general that $\left(_{(\lambda)} \beta\right)$ is a polynomial in $\lambda$.

Example 3. Let $U=U_{0} \oplus U_{1}$ be a finite-dimensional $\mathbb{Z}_{2}$-graded linear space, and let $V=H \otimes U=V_{0} \oplus V_{1}$ be the free $H$-module generated by $U, V_{i}=H \otimes U_{i}$. Then Cend $V$ splits into the sum of even and odd components in a natural way:

$$
(\operatorname{Cend} V)_{0}=\operatorname{Cend}\left(V_{0}\right) \oplus \operatorname{Cend}\left(V_{1}\right), \quad(\operatorname{Cend} V)_{1}=\operatorname{Chom}\left(V_{0}, V_{1}\right) \oplus \operatorname{Chom}\left(V_{1}, V_{0}\right) \text {, }
$$

and the bracket

$$
\left[\alpha_{(\lambda)} \beta\right]=\left(\alpha_{(\lambda)} \beta\right)-(-1)^{|\alpha||\beta|}\left(\beta_{(-\partial-\lambda)} \alpha\right)
$$

turns Cend $V$ into a Lie conformal superalgebra denoted gc $V$.

If $V$ is a free $H$-module of rank $n+m$, where $n=\operatorname{dim} U_{0}, m=\operatorname{dim} U_{1}$, then $\operatorname{gc} V$ is denoted $\mathrm{gc}_{n \mid m}$. This conformal superalgebra may be presented as

$$
H \otimes H \otimes M_{n \mid m}(\mathbb{k}) \simeq H \otimes M_{n \mid m}(\mathbb{k}[x]),
$$

where $M_{n \mid m}$ stands for the $\mathbb{Z}_{2}$-graded algebra of $(n+m)$-matrices with even component $\left(\begin{array}{cc}M_{n} & 0 \\ 0 & M_{m}\end{array}\right)$ and odd component $\left(\begin{array}{cc}0 & M_{n, m} \\ M_{m, n} & 0\end{array}\right)$. The $\lambda$-bracket is given by

$$
[A(x)(\lambda) B(x)]=A(x) B(x+\lambda)-(-1)^{|A||B|} B(x) A(x-\partial-\lambda),
$$

for homogeneous matrices $A, B \in M_{n \mid m}(\mathbb{k}[x])$.

A finite representation of a Lie conformal superalgebra $L$ is a homomorphism of Lie conformal superalgebras

$$
\rho: L \rightarrow \mathrm{gc}_{n \mid m}
$$

In order to have a finite faithful representation (FFR), a Lie conformal superalgebra has to be a torsion-free $H$-module since $\rho\left(\right.$ tor $\left._{\mathrm{H}} L\right)=0$.

Every finite torsion-free Lie conformal superalgebra $L$ has a regular representation on itself: $\rho(a)=\alpha$ for $a \in L$, where $\alpha_{\lambda}(x)=\left[a_{(\lambda)} x\right]$. 
In general, a representation of a Lie conformal superalgebra $L$ on a $\mathbb{Z}_{2}$-graded $H$-module $V=V_{0} \oplus V_{1}$ is a sesqui-linear map $\rho_{\lambda}(\cdot, \cdot): L \otimes V \rightarrow V[\lambda]$ which respects the gradings and

$$
\rho_{\lambda}\left(a, \rho_{\mu}(b, x)\right)-(-1)^{|a||b|} \rho_{\mu}\left(b, \rho_{\lambda}(a, x)\right)=\rho_{\lambda+\mu}\left(\left[a_{(\lambda)} b\right], x\right),
$$

for $a, b \in L_{0} \cup L_{1}, x \in V$.

This is unknown whether all torsion-free finite Lie conformal (super)algebras have FFR. In the non-graded case, it is known [16] that if the solvable radical of $L$ splits then $L$ has a FFR. The proof of the latter result essentially involves representation theory of Lie conformal algebras [8]. For example, if $V$ is the exceptional GD-algebra from Example 1 then $L(V)$ has a split solvable radical. However, a quadratic Lie conformal (super)algebra may have a non-split solvable radical. For example, the Virasoro Lie conformal algebra has a non-split extension [3, Theorem 7.2] corresponding to the 2-dimensional Novikov algebra $V=\mathbb{k} v+\mathbb{k} u$, where

$$
v \circ v=v+u, \quad v \circ u=0, \quad u \circ v=u, \quad u \circ u=0 .
$$

Considered as an Abelian Lie algebra, $V$ is a GD-algebra that gives rise to a quadratic Lie conformal algebra $L(V)$ with a non-split solvable radical $H \otimes \mathbb{k} u$.

In the next section, we will show that for every special GD-superalgebra $V$ the corresponding Lie conformal superalgebra $L(V)$ has a FFR.

\section{Poisson CONFORMAL SUPERALGEBRAS}

In the study of Ado-type problems for Lie conformal algebras, Poisson structures play an important role.

Definition 2. Let $P$ be a $\mathbb{Z}_{2}$-graded $H$-module endowed with two sesqui-linear operations

$$
\begin{aligned}
& {\left[{ }_{(\lambda)} \cdot\right]: P \otimes P \rightarrow P[\lambda],} \\
& \left(\cdot{ }_{(\lambda)} \cdot\right): P \otimes P \rightarrow P[\lambda],
\end{aligned}
$$

which respect the grading and satisfy the following conditions:

(1) $P$ is a Lie conformal superalgebra relative to $\left[\cdot{ }_{(\lambda)} \cdot\right]$;

(2) $\left(a_{(\lambda)}\left(b_{(\mu)} c\right)\right)=\left(\left(_{(\lambda)} b\right)_{(\lambda+\mu)} c\right)$ for $a, b, c \in P$;

(3) $\left(a_{(\lambda)} b\right)=(-1)^{|a||b|}\left(b_{(-\partial-\lambda)} a\right)$ for homogeneous $a, b \in P$.

Then $P$ is said to be a Poisson conformal superalgebra if the following conformal analogue of the Leibniz rule holds:

$$
\left[a_{(\lambda)}\left(b_{(\mu)} c\right)\right]=\left(\left[a_{(\lambda)} b\right]_{(\lambda+\mu)} c\right)+(-1)^{|a||b|}\left(b_{(\mu)}\left[a_{(\lambda)} c\right]\right),
$$

for $a, b \in P_{0} \cup P_{1}, c \in P$.

A simplest example of a Poisson conformal (super)algebra is provided by the current functor. Namely, if $\mathfrak{p}$ is an ordinary commutative (super)algebra with a 
(super)Poisson bracket $\{\cdot, \cdot\}$ then $\operatorname{Cur} \mathfrak{p}=H \otimes \mathfrak{p} \simeq \mathfrak{p}[\partial]$ equipped with

$$
\begin{gathered}
\left(a(\partial)_{(\lambda)} b(\partial)\right)=a(-\lambda) b(\partial+\lambda), \\
{\left[a(\partial)_{(\lambda)} b(\partial)\right]=\{a(-\lambda), b(\partial+\lambda)\}}
\end{gathered}
$$

is a Poisson conformal (super)algebra.

An associative conformal algebra [14] is an $H$-module $A$ equipped with a sesquilinear operation $\left(\cdot{ }_{(\lambda)} \cdot\right)$ satisfying the Condition 2 of Definition 2 , Assuming $A$ is a $\mathbb{Z}_{2}$-graded associative conformal algebra, the new (commutator) operation

$$
\left[a_{(\lambda)} b\right]=\left(a_{(\lambda)} b\right)-(-1)^{|a||b|}\left(b_{(-\partial-\lambda)} a\right), \quad a, b \in A_{0} \cup A_{1},
$$

turns the $H$-module $A$ into a Lie conformal superalgebra $A^{(-)}$(see [23, p. 323] for non-graded case).

Given a Lie conformal superalgebra $L$ and a $\mathbb{Z}_{2}$-graded associative conformal algebra $A$, we say $A$ is an associative conformal envelope of $L$ if there exists a homogeneous homomorphism $\tau: L \rightarrow A^{(-)}$of conformal algebras such that $A$ is generated (as an associative conformal algebra) by the image of $L$. For a fixed $L$, there exists a lattice of universal associative conformal envelopes of $L$ corresponding to different associative locality bounds on the elements of $L$ (see [23, Section 6]). It may happen that neither of these universal envelopes contains the isomorphic image of $L$, i.e., there exist Lie conformal (super)algebras that cannot be embedded into associative ones.

Suppose $A$ is an associative conformal envelope of a Lie conformal superalgebra $L$. Then $A$ has a natural filtration as an $H$-module:

$$
0=A_{0} \subset A_{1} \subset A_{2} \subset \ldots,
$$

where $A_{1}=\tau(L), A_{n+1}=A_{n}+H\left\{\left.\left(\tau(L)_{(\lambda)} A_{n}\right)\right|_{\lambda=\alpha}: \alpha \in \mathbb{k}\right\}$. Then

$$
\left(A_{n(\lambda)} A_{m}\right) \subseteq A_{n+m}[\lambda], \quad\left[A_{n}(\lambda) A_{m}\right] \subseteq A_{n+m-1}[\lambda]
$$

so the associated graded $H$-module gr $A$ has a well-defined structure of a Poisson conformal superalgebra.

For example, conformal Weyl algebra Cend $_{1, x}$ (see [6]) is an associative envelope of the Virasoro conformal algebra. The corresponding Poisson conformal algebra $\operatorname{gr} \operatorname{Cend}_{1, x} \simeq \mathbb{k}[\partial] \otimes x \mathbb{k}[x]$ has the following operations:

$$
\left(x_{(\lambda)}^{n} x^{m}\right)=x^{n+m}, \quad\left[x_{(\lambda)}^{n} x^{m}\right]=(n \partial+(n+m) \lambda) x^{n+m-1} .
$$

More examples of Poisson conformal superalgebra structures on universal associative envelopes of Lie conformal superalgebras can be found in [18].

Hereinafter, we will need the following example of a Poisson conformal superalgebra.

Lemma 4. Let $\mathfrak{p}=\mathfrak{p}_{0} \oplus \mathfrak{p}_{1}$ be an ordinary Poisson superalgebra equipped with an even derivation $d: a \mapsto a^{\prime}$. The latter means $d\left(\mathfrak{p}_{i}\right) \subseteq \mathfrak{p}_{i}, i=0,1,(a b)^{\prime}=a b^{\prime}+a^{\prime} b$, $\{a, b\}^{\prime}=\left\{a^{\prime}, b\right\}+\left\{a, b^{\prime}\right\}$ for $a, b \in \mathfrak{p}$. Then $L(\mathfrak{p}, d)=H \otimes \mathfrak{p}$ equipped with operations

$$
\left(a_{(\lambda)} b\right)=a b, \quad\left[a_{(\lambda)} b\right]=\{a, b\}+\partial a^{\prime} b+\lambda(a b)^{\prime},
$$


for $a, b \in \mathfrak{p}_{0} \cup \mathfrak{p}_{1}$, is a Poisson conformal superalgebra.

Proof. The operation $\left(\cdot{ }_{(\lambda)} \cdot\right)$ is obviously associative and (super-) commutative. By definition, $\left[\cdot{ }_{(\lambda)} \cdot\right]$ is exactly the quadratic Lie conformal bracket on the GDsuperalgebra obtained from $\mathfrak{p}$ relative to $d$. The Leibniz rule (17) is straightforward to check. On the one hand,

$$
\begin{aligned}
& {\left[a_{(\lambda)}\left(b_{(\mu)} c\right)\right]-(-1)^{|a||b|}\left(b_{(\mu)}\left[a_{(\lambda)} c\right]\right)} \\
& =\{a, b c\}+\partial a^{\prime} b c+\lambda(a b c)^{\prime}-(-1)^{|a||b|}\left(b\{a, c\}+(\partial+\mu) b a^{\prime} c+\lambda b(a c)^{\prime}\right) \\
& =\{a, b\} c-\mu a^{\prime} b c+\lambda a b^{\prime} c
\end{aligned}
$$

for homogeneous $a, b, c \in \mathfrak{p}$. On the other hand,

$$
\left(\left[a_{(\lambda)} b\right]_{(\lambda+\mu)} c\right)=\{a, b\} c-(\lambda+\mu) a^{\prime} b c+\lambda(a b)^{\prime} c=\{a, b\} c-\mu a^{\prime} b c+\lambda a b^{\prime} c .
$$

Hence, $L(\mathfrak{p}, d)$ is a Poisson conformal superalgebra.

Suppose $L$ is a Lie conformal superalgebra with a representation $\rho$ on an $H$ module $V$. Then a deformation of $\rho$ is a representation $\rho_{\varepsilon}$ of $L$ on the $H$-module $V \oplus V \varepsilon \simeq \mathbb{k}[\varepsilon] \otimes V /\left(\varepsilon^{2}\right)$, where

$$
\rho_{\varepsilon}(a)=\alpha^{(\varepsilon)}, \quad \alpha_{\lambda}^{(\varepsilon)}(x)=\alpha_{\lambda}(x)+\varepsilon \varphi_{\lambda}(a, x),
$$

for $a \in L, x \in V, \alpha=\rho(a)$. Here $\varphi_{\lambda}(\cdot, \cdot): L \otimes V \rightarrow V[\lambda]$ is a sesqui-linear map which has to satisfy the following equation (a consequence of the Jacobi identity (16)) ):

$$
\begin{aligned}
\varphi_{\lambda+\mu}\left(\left[a_{(\lambda)} b\right], x\right)=\varphi_{\lambda}\left(a, \rho_{\mu}(b, x)\right)+\rho_{\lambda}\left(a, \varphi_{\mu}(b, x)\right) & \\
& -(-1)^{|a||b|} \varphi_{\mu}\left(b, \rho_{\lambda}(a, x)\right)-(-1)^{|a||b|} \rho_{\mu}\left(b, \varphi_{\lambda}(a, x)\right),
\end{aligned}
$$

for $a, b \in L_{0} \cup L_{1}, x \in V$. In the case when $V$ is a finitely generated $H$-module, the relation (18) exactly means that $\varphi$ is a 1-cocycle in $Z^{1}(L$, Cend $V$ ) (see [3]).

Proposition 1. Let $L$ be a graded Lie conformal subalgebra in a Poisson conformal superalgebra $P$. Then $L$ has a regular representation on $P$, and the sesqui-linear $\operatorname{map} \varphi_{\lambda}(\cdot, \cdot): L \otimes P \rightarrow P[\lambda]$ given by

$$
\varphi_{\lambda}(a, x)=\lambda\left(a_{(\lambda)} x\right), \quad a \in L, x \in P,
$$

satisfies (18).

Proof. Obviously, the map $\rho_{\lambda}(a, x)=\left[a_{(\lambda)} x\right], a \in L, x \in P$, is a representation of $L$ on $P$. Then the right-hand side of (18) can be transformed by (17) as

$$
\begin{aligned}
\lambda\left(a_{(\lambda)}\left[b_{(\mu)} x\right]\right) & +\mu\left[a_{(\lambda)}\left(b_{(\mu)} x\right)\right] \\
- & (-1)^{|a||b|} \mu\left(b_{(\mu)}\left[a_{(\lambda)} x\right]\right)-(-1)^{|a||b|} \lambda\left[b_{(\mu)}\left(a_{(\lambda)} x\right)\right] \\
& =\lambda\left(\left[a_{(\lambda)} b\right]_{(\lambda+\mu)} x\right)+\mu\left(\left[a_{(\lambda)} b\right]_{(\lambda+\mu)} x\right)=\varphi_{\lambda+\mu}\left(\left[a_{(\lambda)} b\right], x\right) .
\end{aligned}
$$


Corollary 1 ([18]). Let $L$ be a graded Lie conformal subalgebra of a Poisson conformal superalgebra. Then the map

$$
\hat{\rho}_{\lambda}(a, x)=\left[a_{(\lambda)} x\right]+\lambda\left(a_{(\lambda)} x\right), \quad a \in L, x \in P,
$$

is a representation of $L$ on $P$.

Proof. In order to check the Jacobi identity (16) for $\hat{\rho}$, note that the desired equation

$$
\hat{\rho}_{\lambda}\left(a, \hat{\rho}_{\mu}(b, x)\right)-(-1)^{|a||b|} \hat{\rho}_{\mu}\left(b, \hat{\rho}_{\lambda}(a, x)\right)=\hat{\rho}_{\lambda+\mu}\left(\left[a_{(\lambda)} b\right], x\right)
$$

splits into three equations: the first one is exactly the Jacobi identity for regular representation $\rho_{\lambda}(a, x)=\left[a_{(\lambda)} x\right]$, the second one is (18) for $\varphi_{\lambda}(a, x)=\lambda\left(a_{(\lambda)} x\right)$, and the third one is

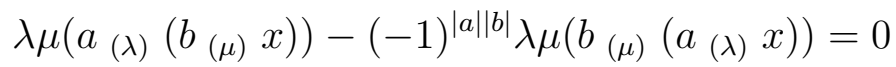

which also holds due to conformal commutativity and associativity of $(\cdot(\lambda) \cdot)$ :

$$
\begin{aligned}
\left(a_{(\lambda)}\left(b_{(\mu)} x\right)\right)=\left(\left(a_{(\lambda)} b\right)_{(\mu+\lambda)} x\right)=(-1)^{|a||b|}\left(\left(b_{(-\partial-\lambda)} a\right)_{(\lambda+\mu)} x\right)^{|a||b|}\left(\left(b_{(\mu)} a\right)_{(\lambda+\mu)} x\right)=(-1)^{|a||b|}\left(b_{(\mu)}\left(a_{(\lambda)} x\right)\right) . \\
=(-1)
\end{aligned}
$$

Corollary 2. If for a finite Lie conformal superalgebra $L$ there exists an embedding $\tau: L \rightarrow P$ of $L$ into a Poisson conformal superalgebra $P$ in such a way that $(\tau(a)(\lambda)$ $\tau(L)) \neq 0$ for all $0 \neq a \in L$ then $L$ has a FFR.

Proof. Indeed, if we consider $V=L$ as a regular $L$-module, $M=\tau(L)+\mathbb{k}[\partial] \otimes$ $\operatorname{span}\left\{\left.\left(\tau(L){ }_{(\lambda)} \tau(L)\right)\right|_{\lambda=\alpha}: \alpha \in \mathbb{k}\right\}$ as an $L$-submodule of $P$, and

$$
\langle\cdot(\lambda) \cdot\rangle: L \otimes V \rightarrow M[\lambda]
$$

given by $\left\langle a_{(\lambda)} b\right\rangle=\left(\tau(a)_{(\lambda)} \tau(b)\right)$, then all conditions of [15, Theorem 3] hold and $L$ has a FFR.

In particular, if $L$ satisfies the Poincaré-Birkhoff-Witt condition [23] then one may choose $P$ to be the associated graded conformal algebra of the appropriate universal associative conformal envelope of $L$. Therefore, for conformal algebras the PBW theorem implies the Ado Theorem immediately [15].

Theorem 3. Let $V$ be a finite-dimensional special GD-superalgebra. Then the Lie conformal superalgebra $L(V)$ has a finite faithful conformal representation.

Proof. Let us fix linear bases $X_{0}$ and $X_{1}$ of $V_{0}$ and $V_{1}$, respectively, and let $X=$ $X_{0} \cup X_{1}$. A special GD-superalgebra embeds into its universal enveloping differential Poisson superalgebra which can be constructed as follows. Denote by $F=s$ PoisDer $\left\langle X_{0} \cup X_{1}, d\right\rangle$ the free differential Poisson superalgebra with an identity element 1 (generated by even elements $X_{0}$, odd elements $X_{1}$ ) and an even derivation $d$. Let $I_{V}$ stand for the (differential) ideal of $F$ generated by

$$
x d(y)-x \circ y, \quad x, y \in X,
$$


where $x \circ y$ is a linear form in $\mathbb{k} X$ representing the Novikov product in $V$. The quotient $F / I_{V}$ is the universal enveloping differential Poisson superalgebra for $V$ denoted $P_{d}(V)$. If $V$ is a special GD-superalgebra then $V$ embeds into $P_{d}(V)$.

The free algebra $F$ may be presented as

$$
F=\bigoplus_{n \in \mathbb{Z}} F_{n}
$$

where $F_{n}$ consists of all elements of weight $n$. Recall that the weight in a free differential Poisson (super)algebra generated by a set $X$ is defined as follows [17]:

$$
\begin{gathered}
\text { wt } x=-1 \text { for } x \in X, \quad \operatorname{wt}(1)=0, \\
\operatorname{wt}(u v)=\operatorname{wt} u+\operatorname{wt} v, \quad \operatorname{wt}\{u, v\}=\operatorname{wt} u+\operatorname{wt} v+1, \\
\operatorname{wt} d(u)=\operatorname{wt} u+1 .
\end{gathered}
$$

Since all elements in (20) are wt-homogeneous, the ideal $I_{V}$ is wt-homogeneous and the algebra $P_{d}(V)$ inherits the grading:

$$
P_{d}(V)=\bigoplus_{n \in \mathbb{Z}} U_{n}, \quad U_{n}=F_{n} / I_{V} \cap F_{n}
$$

Note that $V \simeq U_{-1}$. The latter was shown in [17, Theorem 10] for non-graded case, the same reasonings work for superalgebras.

Lemma 4 states that $L\left(P_{d}(V), d\right)=H \otimes P_{d}(V)$ is a Poisson conformal superalgebra. Then by Corollary 1 the Lie conformal superalgebra $L=L(V)$ has a representation on $M=L\left(P_{d}(V), d\right)$ given by

$$
\hat{\rho}_{\lambda}(a, u)=\{a, u\}+\partial d(a) u+\lambda(d(a u)+a u), \quad a \in V, u \in P_{d}(V) .
$$

Obviously, for every $m \in \mathbb{Z}$ the space $M_{\leq m}=H \otimes \bigoplus_{n \leq m} U_{n}$ is a conformal $L$ submodule of $M$. In particular, $\bar{M}=M_{\leq 0} / M_{\leq-2} \simeq H \otimes\left(U_{-1} \oplus U_{0}\right)$ is a conformal $L$ module corresponding to a representation $\bar{\rho}$ defined by (21) for $u \in U_{0}$ and $\bar{\rho}_{\lambda}(a, u)=$ $\left[a_{(\lambda)} u\right]$ for $u \in U_{-1} \simeq V$. It is easy to see that the representation of $L$ on $\bar{M}$ is faithful: $\bar{\rho}_{\lambda}(a, 1)=(\partial+\lambda) d(a)+\lambda a \neq 0$ for $a \in V, a \neq 0$. However, it is not yet finite in general since $\operatorname{dim} U_{0}$ may not be finite.

Note that for every $a \in V \simeq U_{-1}$ the map $\mu_{a}: u \mapsto a u$ maps $U_{0}$ to $U_{-1}$. Since $\operatorname{dim} U_{-1}=\operatorname{dim} V<\infty$, the intersection of all Ker $\mu_{a}, a \in V$, is a subspace of finite codimension. So, consider $N=\left\{u \in U_{0} \mid V u=0\right\}$. For every $u \in N$ we have

$$
\bar{\rho}_{\lambda}(a, u)=\{a, u\}+\partial d(a) u, \quad a \in V .
$$

Given $b \in V, b\{a, u\}=(-1)^{|a||b|}(\{a, b u\}+\{a, b\} u)=0, b d(a) u=(-1)^{|a||b|} d(a) b u=$ 0 . Therefore, $H \otimes N$ is a conformal $L$-submodule of $\bar{M}$. Finally,

$$
\bar{M} / N \simeq H \otimes\left(U_{-1} \oplus U_{0} / N\right)
$$

is a finite faithful conformal $L$-module. 
Corollary 3. If a GD-(super)algebra $V$ is constructed from a Novikov (super)algebra with respect to the commutator then the corresponding quadratic Lie conformal superalgebra $L(V)$ has a FFR.

\section{REFERENCES}

[1] C. Bai, D. Meng, The classification of Novikov algebras in low dimensions, J. Phys. A, Math. Gen. 34 (2001) (8) 1581-1594.

[2] B. Bakalov, A. D'Andrea, V. G. Kac, Theory of finite pseudoalgebras, Adv. Math. 162 (2001) $1-140$.

[3] B. Bakalov, V. G. Kac, A. Voronov, Cohomology of conformal algebras, Comm. Math. Phys. 200 (1999) 561-589.

[4] A. A. Balinskii, S. P. Novikov, Poisson brackets of hydrodynamic type, Frobenius algebras and Lie algebras, Sov. Math. Dokl. 32 (1985) 228-231.

[5] L.A. Bokut, Y. Chen, Z. Zhang, Gröbner-Shirshov bases method for Gelfand-DorfmanNovikov algebras, J. Algebra Appl. 16 (1) (2017) 22.

[6] C. Boyallian, V. G. Kac, J.-I. Liberati, On the classification of subalgebras of Cend ${ }_{N}$ and $\mathrm{gc}_{N}$. J. Algebra 260 (2003) 32-63.

[7] D. Burde, W. de Graaf, Classification of Novikov algebras, Appl. Algebra Eng. Commun. Comput. 24 (2013) (1) 1-15.

[8] S.-J. Cheng, V. G. Kac, Conformal modules, Asian J. Math. 1 (1997) 181-193.

[9] A. S. Dzhumadil'daev, C. Löfwall, Trees, free right-symmetric algebras, free Novikov algebras and identities, Homology, Homotopy Appl. 4 (2002) (2) 165-190.

[10] Li Guo, W. Keigher, On differential Rota-Baxter algebras, J. Pure Appl. Algebra 212 (2008) (3) $522-540$.

[11] I. M. Gelfand, I. Ya. Dorfman, Hamilton operators and associated algebraic structures, Functional analysis and its application 13 (1979) (4) 13-30.

[12] V. Ginzburg, M. Kapranov, Koszul duality for operads, Duke Math. J. 76 (1994) (1) 203-272.

[13] Y. Hong, Z. Wu, Simplicity of quadratic Lie conformal algebras, Comm. Algebra 45 (2017) (1) $141-150$.

[14] V. G. Kac, Vertex Algebras for Beginners. University Lecture Series, 10, 2nd edn. American Mathematical Society, Providence, 1996 (1998).

[15] P. S. Kolesnikov, On finite representations of conformal algebras. J. Algebra 331 (2011) 169193.

[16] P. S. Kolesnikov, The Ado theorem for finite Lie conformal algebras with Levi decomposition. J. Algebra Appl. 15 (2016) Art.no. 1650130.

[17] P. S. Kolesnikov, B. Sartayev, A. Orazgaliev, Gelfand-Dorfman algebras, derived identities, and the Manin product of operads, Journal of Algebra 539 (2019) 260-284.

[18] P. S. Kolesnikov, Universal enveloping Poisson conformal algebras, Internat. J. Algebra Comput. 30 (2020) no. 5, 1015-1034.

[19] J.-L. Loday, On the operad of associative algebras with derivation, Georgian Math. J. 17 (2010) (2) 347-372.

[20] J.-L. Loday, B. Vallette, Algebraic Operads, Gründlehren der mathematischen Wissenschaften 346, Springer-Verlag, 2012.

[21] A. A. Mikhalev, I. P. Shestakov, PBW-pairs of varieties of linear algebras, Comm. Algebra 42 (2014) 667-687.

[22] J. M. Osborn, Novikov algebras, Nova J. Algebra \& Geom. 1 (1992) 1-14.

[23] M. Roitman, Universal enveloping conformal algebras, Sel. Math., New Ser. 6 (2000) 319-345.

[24] I. R. Shafarevich, Degeneration of semisimple algebras, Comm. Algebra 29 (2001) no. 9, 3943-3960. 
[25] A. I. Shirshov, On free Lie rings, Mat. Sb., 45(87) (1958) 113-122.

[26] X. Xu, On simple Novikov algebras and their irreducible modules, J. Algebra 185 (1996) (3) 905-934.

[27] X. Xu, Quadratic Conformal Superalgebras, J. Algebra 231 (2000) 1-38.

[28] X. Xu, Classification of simple Novikov algebras and their irreducible modules of characteristic 0, J. Algebra 246, (2001) (2) 673-707.

[29] X. Xu, Gel'fand-Dorfman bialgebras, Southeast Asian Bull. Math. 27 (2003) 561-574.

[30] E. I. Zel'manov, On a class of local translation invariant Lie algebras, Sov. Math. Dokl. 35 (1987) 216-218.

[31] Z. Zhang, Y. Chen, L.A. Bokut, Free Gelfand-Dorfman-Novikov superalgebras and a Poincaré-Birkhoff-Witt type theorem, Int. J. Algebra Comput. 29 (2019), no. 3, 481-505.

1) Sobolev Institute of Mathematics, Novosibirsk, Russia

2) Novosibirsk State University, Novosibirsk, Russia 